\title{
Escala de Competencias Socioemocionales
}

\author{
Scale of Socio-emotional Competences
}

Kiara Shalom Rojas Aliga1*, Luz Nayeli Huamaní Miguel², Linsey Wildman Vilca Quiro³

\begin{abstract}
RESUMEN
Objetivo: Determinar las propiedades psicométricas de la Escala de Competencias Socioemocionales (ECSE) en trabajadores de instituciones públicas y privadas de Lima Este. Material y métodos: El estudio fue de diseño no experimental de carácter transversal, además se caracteriza por ser sistemático, controlado, empírico y crítico. Se utilizó el muestreo no probabilístico, a conveniencia de los investigadores, basado en criterios de inclusión y exclusión previamente establecidos, la muestra estuvo conformada por 186 trabajadores, entre las edades de 21 y 65 años. El proceso de validación de la prueba comprendió un análisis de la validez de contenido a través de la evaluación por jueces y la evaluación psicométrica realizada en una sola fase: muestra piloto de validación para determinar la confiabilidad de la prueba a través del análisis de dificultad de los ítems, la correlación ítem-test, la estimación del coeficiente de Cronbach y el análisis factorial. Resultados: Como resultado del análisis factorial exploratorio, se demostró que el constructo está conformado por 2 dimensiones, competencia emocional y social, con un 44.87 \% de la varianza total y a su vez por 5 subdimensiones (conocimiento emocional, regulación emocional, capacidad participativa, empatía y resolución de conflictos); el coeficiente $\alpha$ de Cronbach para el instrumento en su conjunto fue de.793; asimismo, se retuvo aquellos ítems que cumplan el siguiente requerimiento a) correlación ítem - subdimensión mayor a 0.3 , b) coeficiente alpha de crombach (al eliminar el ítem) igual o menor de la subdimensión, c) saturación factorial en su dimensión por encima de 0.30. Como resultado de este análisis fueron invalidados 31 ítems, los cuales fueron eliminados, quedando constituido el instrumento por 28 ítems. Conclusiones: El instrumento posee propiedades psicométricas de validez de constructo, así como un buen nivel de confiabilidad.
\end{abstract}

Palabras clave: Competencia socioemocional, fiabilidad, validez de constructo, consistencia interna.

\begin{abstract}
Objective: To determine the psychometric properties of the scale of socio-emotional skills (ECSE) for workers in public and private institutions in Lima Este. Maethods: The study was not experimental transversal design, further characterized by systematic, controlled, empirical and critical. Non-probability sampling was used for the convenience of researchers, based on criteria previously established inclusion and exclusion, the sample consisted of 186 workers, between the ages of 21 and 65. The validation process of the test comprised an analysis of content validity by judges and evaluation by psychometric assessment in one phase: pilot validation sample to determine the reliability of the test through the analysis of difficulty items, item-test correlation, estimation of Cronbach and factor analysis. Results: As a result of exploratory factor analysis showed that the construct is comprised of 2-dimensional competition emotional and social with $44.87 \%$ of the total variance and in turn by 5 sub dimensions (emotional awareness, emotional regulation, participatory capacity, empathy and dispute resolution); Cronbach's $\alpha$ for the whole instrument was 0.793 ; also those items that meet the following requirements a) correlation was retained item - larger sub $0.3, \mathrm{~b}$ ) Cronbach alpha coefficient (to remove the item) equal to or less than the sub dimension, c) in its dimension factorial saturation above 0.30 . As a result of this analysis they were invalidated 31 items, which were eliminated, leaving the instrument consisting of 28 items. Conclusion: The instrument has psychometric properties of construct validity as well as a good level of reliability
\end{abstract}

Keywords: Socio-emotional competence, reliability, construct validity, internal consistency.

${ }^{1}$ Psicóloga del Colegio Unión, Lima, Perú

${ }^{2}$ Psicóloga y profesora, Sunshine prep high School, Muzaffarpur, Bihar, El Cairo, Egipto

${ }^{3}$ Docente de la EP de Psicología, Facultad de Ciencias de la Salud, Universidad Peruana Unión, Lima, Perú 


\section{INTRODUCCIÓN}

La globalización presenta un nuevo entorno que tiene relación estrecha con los cambios en las empresas y en la formación de recursos humanos para hacerlas más competitivas, llevándolos a replantear sus estrategias de producción y a la vez su modo de manejar el personal, siendo más exigentes en su selección, concientizando a que los trabajadores aporten no solo conocimiento sino también habilidades, destrezas y características humanas para mejorar la productividad (Quintero, 2004). Por esta razón las empresas, a la hora de gestionar su personal, adoptan la gestión del talento humano por competencias.

Actualmente, en la evaluación de puestos de trabajo, no solo se evalúa el conocimiento y la experiencia sino también las competencias que requiere el puesto (Morales, 2008). De manera similar Cantero (2012) y Pérez (2003) afirman que las competencias socioemocionales tienen una importancia relevante dentro de una organización ya que están relacionadas con la salud mental, desarrollo de la carrera profesional, el desempeño laboral, el liderazgo efectivo, el afrontamiento del estrés laboral y la disminución del nivel de agresividad en las organizaciones. Entonces para mantener buenas relaciones en el trabajo y para lograr un alto desempeño laboral se requieren, además, otras competencias de tipo social y emocional (Cantero, 2012).

En el Perú, Becerra y La Serna (2005) evaluaron a diversas empresas nacionales para analizar las competencias que demanda actualmente el mercado laboral peruano, llegando a la conclusión que las competencias más demandadas incluyen las habilidades para sostener relaciones interpersonales, trabajar en equipo y orientarse al cliente. Además, las empresas requieren personal analítico, proactivo, orientado a resultados y con capacidad para adaptarse a los cambios. Asimismo, Manpower Group (2013) indicó que cada tres empleadores en el mundo no pueden encontrar el talento crítico que requiere para su negocio, al igual que en el Perú el 19\% de profesionales desempleados se debe a la falta de competencias tales como inteligencia emocional, liderazgo y la comunicación efectiva.

La Organización Internacional de Trabajo (OIT, 2013) señala que muchos de los trabajadores que han perdido su empleo a nivel mundial, no es solo por falta de conocimiento, sino también por no poseer aquellas competencias que exige el mercado laboral actual, entre ellas se incluye las competencias socioemocionales. En la misma línea, Vallejo (2005) menciona que la mayoría de los conflictos intra e interpersonales, así como los futuros tropiezos personales y profesionales se suelen deber más a carencias socioemocionales que intelectuales. Entonces, las competencias socioemocionales facilitan la inserción laboral y potencian la empleabilidad, ya que cumplen un rol fundamental dentro del campo laboral, especialmente en las áreas de educación, salud y servicios sociales, donde existe un mayor contacto interpersonal y es necesario el manejo de los escenarios conflictivos (Repetto, Pena, Mudarra, \& Urribari, 2009; Costa, 2003; Cantero, 2012).

No obstante, se evidencia cierta dificultad y ambigüedad en cuanto a su evaluación, ya que diferentes autores utilizan instrumentos que tienen como finalidad medir la inteligencia emocional, evidenciándose una inexactitud en el objetivo de la investigación y lo que se está evaluando, posiblemente por la carencia de instrumentos que midan específicamente este constructo (Molero, Belchi y Torres, 2012; Fernández, 2011; Sainz, et al., 2011 y Cantero, Pérez, Pérez, 2008).

En países tales como España y Estados Unidos se han realizado investigaciones sobre este constructo y su medición en adultos, niños y adolescentes, entre ellos resalta el estudio de Repetto, Beltrán, Garay y Pena (2006), donde construyeron y validaron la escala de Competencias Socioemocionales (ICS-I; ICS.P), con las siguientes dimensiones: autoconciencia emocional, autorregulación emocional, motivación, empatía y competencias sociales. Tambien Cohn, Merrell, Felver-Grant, Tom y Endrulat (2009) validaron el cuestionario SEARS-C (Habilidades socioemocionales y resiliencia en niños) y SEARS-A (Habilidades socioemocionales y resiliencia en adolescentes), teniendo como dimensiones múltiples áreas: habilidades de amistad, empatía, habilidades interpersonales, apoyo social, resolución de problemas, competencia emocional, madurez social, autoconcepto, autogestión, independencia social, estrategias cognitivas y resiliencia socioemocional.

Sin embargo, con respecto a la realidad peruana, no se encuentran instrumentos que midan este constructo, siendo evidente la importancia de tener instrumentos fiables y válidos que evalúen esta problemática.

\section{Conceptualización del constructo Competencias socioemocionales}

Para Goleman (1995, 1999 y 2001) la inteligencia emocional está compuesta por dos tipos de competencias: personales (conciencia de uno mismo, autorregulación y motivación) y sociales (empatía 
y habilidades sociales) dichas competencias son capacidades adquiridas (aprendizaje).

Más adelante, Costa (2003) señala que las competencias socioemocionales son comportamientos sociales/interpersonales que resultan efectivos en la obtención de resultados positivos del entorno, las cuales se observan en el trato fácil con el entorno, facilidad de hacer amigos, afrontar sin dificultad las relaciones y conflictos que surgen en el entorno, utilizar una conversación fluida, expresar puntos de vista y desacuerdos sin que los demás se sientan atacados, saber decir no y rechazar ofrecimientos que resultan peligrosos sin que se sienta menospreciado el entorno, saber llegar acuerdos, tolerar bien el que exista desacuerdos, atreverse a mantener opciones diferentes a las de los demás, valorar positivamente y ser respetuoso con las opciones diferentes a las suyas.

Por otro lado, Repetto, Beltrán, Garay y Pena (2006) mencionan que es un conjunto de conductas de contenido emocional y social que se dan en diferentes contextos y situaciones laborales, aportando calidad y eficacia en el desarrollo profesional y basados en las clasificaciones de diversos autores (Bar-On, 1997; Goleman, 1999; Mayer y Salovey,1997 y Saarni, 2000) donde afirman que las competencias socioemocionales están compuestas por 5 subcompetencias: autoconciencia emocional, autorregulación, empatía, motivación y competencias sociales. Bar-On (1997) refiere que las personas que poseen competencias socioemocionales, se caracterizan por contar con habilidades interpersonales como: el de estar atento a sí mismo, entender las fortalezas y las debilidades, expresar sentimientos y pensamientos de manera no destructiva. Por último, Gonzáles (2014) las consideraba como un conjunto de habilidades que permite la relación eficaz con el medio y con uno mismo, reconociendo, manejando y expresando correctamente las emociones.

A partir de la revisión literaria, se define a las competencias socioemocionales como la capacidad para comprender, regular y expresar nuestras emociones, basadas en un procesamiento cognitivoafectivo que favorezcan a una mayor adaptación en el contexto social, promoviendo el bienestar intra e interpersonal.

Por lo expuesto, el estudio pretende construir y validar un instrumento que evalúe las competencias socioemocionales en trabajadores de distintas profesiones de Lima Este.

\section{MATERIAL Y MÉTODOS}

La población considerada, para la construcción y adaptación del instrumento, estuvo conformada por trabajadores quienes laboran en instituciones privadas y públicas de Lima Este. La construcción de la escala consta de tres etapas: (1) definición conceptual de la construcción y el desarrollo de los temas, (2) el desarrollo de la escala y (3) la confirmación de las propiedades psicométricas.

\section{Participantes}

La muestra piloto fue de 186 trabajadores, compuesta por 102 varones y 84 mujeres de instituciones públicas y privadas de Lima Este. Esta muestra se realizó con el fin de construir el instrumento de medición.

\section{Procedimiento y análisis de datos}

Primera etapa: Definición conceptual de la construcción y el desarrollo de los temas.

Es importante comenzar con una definición completa y rigurosa del constructo a medir, con el propósito de construir un instrumento con las garantías psicométricas necesarias. El marco teórico que especifica las relaciones entre el constructor y sus indicadores (Piñuel, 2001; González y Rodríguez, 2005; Leymann, 1996). Además, la definición de competencias socieomocionales, dimensiones y subdimensiones se basan en el contexto teórico de diversos autores (Goleman, 2001; Repetto y Pérez, 2007; Bar On, 1997; Repetto y Pena, 2010; Bisquerra y Pérez, 2007; Stoner, 1996; Bunk, 1994; Alcañiz y Pérez, 2008; Alles, 2008; Marcuello, 1999; Bisquerra, 2002). Se elaboraron 60 ítems que evaluaron 2 dimensiones (competencia emocional, competencia social) y 5 subdimensiones (conocimiento emocional, regulación emocional, capacidad participativa, empatía y solución de conflictos). Sin embargo, después de realizar un análisis estadístico, se redujo a 28 ítems con 5 subdimensiones.

\section{Segunda etapa: Desarrollo de la escala}

\section{Revisión por criterio de jueces}

Se realizó este proceso, con el fin de garantizar la construcción del instrumento en ambas partes del proceso, es decir, en el contenido y la estructura; estos fueron sometidos a un comité de 5 expertos, formados por dos doctores uno en Psicología Organizacional y otro en Psicología Clínica; tres especialistas en Psicología Organizacional. Todos ellos reconocidos 
por su trayectoria académica a nivel universitario, posgrado e investigación.

Seguidamente los ítems, de la escala, fueron evaluados en base a cuatro criterios: claridad, consistencia, contexto y dominio del constructo. Respecto a la cuantificación, se utilizó el coeficiente $\mathrm{V}$ de Aiken, ya que este coeficiente puede obtener valores que van de 0 a 1; considerando que la validez de contenido es cuanto más cerca de 1, indica que el ítem es adecuado para medir el constructo (Abad, et al, 2006).

\section{Análisis de confiabilidad}

Para la fiabilidad de la escala, se evaluó por medio del método de consistencia interna, para lo cual se utilizó el coeficiente alpha de cronbach, en la muestra piloto. Mediante este proceso se realiza la fiabilidad del test basado en el número de elementos y la proporción de la varianza total de la prueba debido a la covarianza entre los elementos ya que, si es más la covarianza entre los elementos, mayor será la fiabilidad del instrumento.

\section{RESULTADOS}

Tabla 1

Análisis de contenido de instrumento

\begin{tabular}{ll}
\hline \multicolumn{1}{c}{ Test } & V \\
\hline Forma correcta de aplicación y estructura & 1 \\
Orden de las preguntas establecidas adecuadamente & 1 \\
Contiene el test de preguntas difíciles de entender & 1 \\
Contiene el test de palabras difíciles de entender & 1 \\
Las opciones de respuesta son pertinentes y están suficientemente & 1 \\
graduadas & 1 \\
\hline \multicolumn{2}{c}{ Los ítems tienen correspondencia con la dimensión a la que pertenecen 5} \\
\hline
\end{tabular}

La Tabla 1 indica que considerando que la mayoría de los ítems presentan óptimos valores superiores a .80 en la forma correcta de aplicación y estructura, orden de las presuntas establecidas adecuadamente, palabras difíciles de entender, las opiniones de respuestas son pertinentes y están suficientemente graduadas, lo cual proporciona la validez de contenido del instrumento.

Tabla 2

Estimaciones de las dimensiones de la escala CSE

\begin{tabular}{ccc}
\hline Dimensión & $\mathrm{N}^{0}$ de ítems & Alfa \\
\hline Competencia emocional & 12 &, 532 \\
Conciencia emocional & 6 &, 709 \\
Regulación emocional & 6 &, 603 \\
Competencia social & 16 &, 795 \\
Capacidad participativa & 6 &, 729 \\
Empatía & 4 &, 497 \\
Resolución de conflictos & 6 &, 727 \\
Escala global & 28 &, 789
\end{tabular}

En la Tabla 2 se observa que la consistencia interna global de la escala de competencias socioemocionales es adecuada (,789). De manera similar ocurre con la mayoría de las dimensiones y subdimensiones, sin embargo, se aprecia que la subdimensión empatía presenta una baja fiabilidad $(, 497)$ lo que podría indicar que no todos los ítems están evaluando lo mismo. 
Tabla 3

Análisis estadísticos de los ítems

\begin{tabular}{|c|c|c|c|}
\hline Ítems & Correlación & & Alpha \\
\hline & \multicolumn{3}{|c|}{$\begin{array}{c}\text { Correlación ítems test/ alfa si se } \\
\text { elimina el ítem }\end{array}$} \\
\hline \multicolumn{2}{|c|}{ D1 Conocimiento emocional } & ,676 & \\
\hline CE_1 &, $531^{\star *}$ & ,355 & 649 \\
\hline CE_2 &, $503^{* *}$ & ,368 & ,649 \\
\hline CE_3 &, $534^{* *}$ & ,348 & 651 \\
\hline CE_4 &, $521^{\star *}$ & ,381 & ,646 \\
\hline CE_5 & $611^{* *}$ & ,423 & 634 \\
\hline CE_6 &, $552^{* *}$ & ,357 & 649 \\
\hline CE_7 &, $433^{* *}$ & ,269 & ,664 \\
\hline CE_8 &, $426^{* *}$ & ,253 & ,668 \\
\hline CE_9 &, $489 * *$ & ,329 & ,654 \\
\hline CE_10 &, $448^{* *}$ & ,298 & ,660 \\
\hline \multicolumn{2}{|c|}{ D2 Regulación emocional } & ,617 & \\
\hline $\mathrm{RE} \_34$ & $650^{* *}$ & ,413 &, 555 \\
\hline RE_35 &, $305^{\star *}$ & ,171 & 615 \\
\hline RE_36 &, $460 * *$ & ,206 & ,620 \\
\hline RE_37 &, $526^{\star *}$ & ,322 &, 583 \\
\hline RE_38 & $498 * *$ & ,372 &, 581 \\
\hline RE_39 &, $524^{\star *}$ & ,388 &, 575 \\
\hline RE_40 &, $500^{* *}$ & ,312 &, 586 \\
\hline $\mathrm{RE} \_41$ &, $481^{* *}$ & ,283 &, 593 \\
\hline RE_42 &, $530 * *$ & ,315 &, 585 \\
\hline \multicolumn{2}{|c|}{ D 3 Capacidad participativa } & ,774 & \\
\hline CP_43 &, $439 * *$ & ,272 & ,776 \\
\hline CP_44 &, $364^{* *}$ & ,236 & ,774 \\
\hline CP_45 &, $523^{* *}$ & ,445 & ,759 \\
\hline CP_46 &, $440 * *$ & ,368 &, 765 \\
\hline CP_11 &, $418^{* *}$ & ,239 & ,782 \\
\hline CP_12 &, $510^{* *}$ & ,399 & ,760 \\
\hline CP_13 &, $538^{* *}$ & ,465 & ,758 \\
\hline CP_14 &, $479 * *$ & 341 &, 766 \\
\hline CP_15 &, $534^{* *}$ & ,424 &, 758 \\
\hline CP_16 &, $524^{* *}$ & ,449 & ,759 \\
\hline CP_17 &, $567^{\star *}$ & ,466 &, 754 \\
\hline
\end{tabular}

$\begin{array}{llll}\text { CP_18 } & , 619^{* *} & , 554 & , 752 \\ \text { CP_19 } & , 569^{* *} & , 482 & , 754 \\ \text { CP_20 } & , 568^{* *} & , 449 & , 755 \\ \text { CP 21 } & , 571^{* *} & , 452 & , 755\end{array}$

D 4 Empatía

$, 571^{* *} \quad, 452 \quad, 755$

,428

E_22 ,298** , 167 , 408

E_23 , 186 $^{*} \quad-, 042 \quad, 465$

E_24 ,375** ,21 ,393

E_25 ,332** , 180 ,403

E_26 ,376** , 198

E_55 ,421** , 078 ,455

E_56 , 116 , 020 ,432

E_57 , 424** , 239 , 383

E_58 ,548** , 213

E_59 , 104 , 049

E_47 ,393** ,228 ,390

E_48 ,333** , 180 , 403

E_49 ,348** , 256 , 401

E_50 ,289** , 101 ,421

E_51 ,388

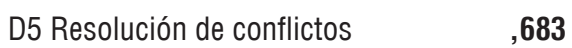

RC_27 ,617** , 468 ,635

RC_28 ,549** , 397 ,650

RC_29 ,659** , 521

RC_30 ,356** , 114 ,714

RC_31 ,607** , 483 ,637

RC_32 , 398** , 201 , 687

RC_33 ,610** ,637

RC_52 ,547** , 363 ,656

RC_53 , 444** , 299 ,667

RC_54 ,399** , 232 ,678

En la Tabla 3 se muestra el análisis de fiabilidad realizado nuevamente, este análisis de los ítems en la segunda etapa simultáneamente se encontró que solo el ítem 4 no cumple con los dos criterios de evaluación y no presenta un adecuado nivel de fiabilidad, ya que está por debajo de un umbral mínimo de .70 (Nunnally, 1978). 
Tabla 4

Matriz de factores rotados para los 5 constructos

\begin{tabular}{|c|c|c|c|c|c|c|}
\hline Ítems & Dimensión & $\mathrm{CP}$ & CE & $\mathrm{RC}$ & $E$ & $\mathrm{RE}$ \\
\hline 16 & $\mathrm{CP}$ & 815 & & & & \\
\hline 18 & $\mathrm{CP}$ & ,718 & & & & \\
\hline 15 & $\mathrm{CP}$ & ,611 & & & & \\
\hline 19 & $\mathrm{CP}$ &, 585 & & & ,326 & \\
\hline 38 & RE &, 511 & & & & ,443 \\
\hline 5 & CE & & ,652 & & & \\
\hline 6 & CE & &, 646 & & & \\
\hline 4 & CE & & ,633 & & & \\
\hline 1 & CE & & ,614 & & & \\
\hline 3 & CE & & ,603 & & & \\
\hline 2 & CE & &, 567 & & & \\
\hline 27 & $\mathrm{RC}$ & & & ,738 & & \\
\hline 28 & $\mathrm{RC}$ & & & ,692 & & \\
\hline 29 & $\mathrm{RC}$ & & & 609 & & \\
\hline 31 & $\mathrm{RC}$ & & & ,598 & ,361 & \\
\hline 33 & $\mathrm{RC}$ & & &, 557 & & \\
\hline 48 & $E$ & & & & 611 & \\
\hline 47 & $E$ & & & &, 598 & \\
\hline 21 & $\mathrm{CP}$ & ,391 & & &, 535 & \\
\hline 51 & $E$ & & & & ,492 & \\
\hline 49 & $\mathrm{E}$ & & & & ,462 & \\
\hline 20 & $\mathrm{CP}$ & ,306 & & ,309 & ,428 & \\
\hline 39 & $\mathrm{RE}$ & & & & & ,636 \\
\hline 34 & RE & & & & & ,602 \\
\hline 37 & $\mathrm{RE}$ & & & & & ,592 \\
\hline 40 & RE & & & & &, 571 \\
\hline 52 & $\mathrm{RC}$ & & & ,344 & ,311 & ,438 \\
\hline 42 & $\mathrm{RE}$ & & & & ,302 & ,420 \\
\hline \multicolumn{2}{|c|}{ \% Total de varianza explicada } & & & $44.87 \%$ & & \\
\hline \multicolumn{2}{|c|}{ Prueba de KMO } & & & ,782 & & \\
\hline \multicolumn{2}{|c|}{ Prueba de Barlett } & & $\left.\right|^{2}(378)$ & $=1196,998$ & $p<.001$ & \\
\hline \multicolumn{2}{|c|}{ \% Total de varianza explicada } & & & $44.87 \%$ & & \\
\hline \multicolumn{2}{|c|}{ Prueba de KMO } & & & ,782 & & \\
\hline \multicolumn{2}{|c|}{ Prueba de Barlett } & & $p^{2}(378)$ & $=1196,998$ & $p<.001$ & \\
\hline
\end{tabular}

Nota: $\mathrm{CP}=$ Competencia Participativa; $\mathrm{CE}=$ Competencia Emocional; $\mathrm{RC}=$ Resolución de Conflictos; $\mathrm{E}=$ Empatía; $\mathrm{RE}=$ Regulación Emocional.

Para evaluar la validez de constructo de la escala de Competencias Socioemocionales, como lo muestra la Tabla 4, se realizó el análisis factorial exploratorio (AFE) para cinco factores fijos utilizando el método de rotación varimax. Se verificó el cumplimiento de los supuestos para realizar el AFE como la prueba de Medida de adecuación muestral Kaiser-Meyer-Olkin (KMO) y la prueba de esfericidad de Bartlett. Se observa que cumple con ambos supuestos. Además, se aprecia que los cinco factores encontrados explican el $44.87 \%$ de toda la varianza y la mayoría de los ítems ingresan en el componente al que le corresponde con un peso factorial alto. Sin embargo, no ocurre 
con todos los ítems, como el 38 que ingresa a una dimensión que teóricamente no le corresponde (competencia participativa), no obstante, tiene peso factorial considerable en su dimensión, por tanto, se toma la decisión de mantener el ítem en su dimensión original. También el ítem 21 y 20 ingresa a una dimensión que teóricamente no le

Tabla 5

Estructura de la escala de ECSE final corresponde (empatía), sin embargo, tiene peso factorial considerable en su dimensión, por lo que se le mantiene en su misma dimensión. Por último, el ítem 52 ingresa a una dimensión que teóricamente no le corresponde (regulación emocional, pero presenta un peso factorial considerable por lo que se decide mantener en su propia dimensión.

\begin{tabular}{ccccc}
\hline Constructo & Dimensión & Sub- dimensión & $N^{\circ}$ de Ítems & Ítems \\
\hline Competencia Socioemocional & Competencia emocional & Conocimiento emocional & $1,2,3,4,5,6$ & 6 \\
& Regulación emocional & $34,37,38,39,40,42$ & 6 & 6 \\
& Competencia social & Capacidad participativa & $15,16,18,19,20,21$ & 6 \\
Empatía & $47,48,49,51$ & 6 \\
Total de ítems & Resolución de conflictos & $27,28,29,31,33,52$ & \\
& 28 & & \\
\hline
\end{tabular}

En la Tabla 5 se observa la estructura de la escala final, después de haber pasado por diversos procesos como revisiones por expertos, análisis de confiabilidad, análisis factorial exploratorio del instrumento final ECSE.

\section{DISCUSIÓN}

Goleman (1995) popularizó el concepto de inteligencia emocional, revitalizando el interés por las competencias sociales y emocionales (competencias socioemocionales) y se han realizado numerosas investigaciones sobre ambos constructos (Repetto y Pérez, 2007). Sin embargo, ambos constructos tienden a ser más bien complementarios antes que contradictorios en su contenido (Pérez, Petrides, y Furnham, 2005). Existe evidencia empírica acumulada como para afirmar que las competencias socioemocionales coexisten con la inteligencia emocional, dado que esta depende de las competencias socioemocionales para poder medirse, logrando de esta manera un buen ajuste psicosocial y bienestar emocional, incluyendo una mejor salud física y mental (Bisquerra, Extremera y FernándezBerrocal, Schutte, Malouff, Thorsteinsson, Bhullar, y Rooke, Petrides, Pérez-González, y Furnham) (citado por Senra, Pérez-González, y Manzano, 2007; Fragoso, 2015).

Los resultados de la investigación se asemejan a las de otras investigaciones encontradas como la de Repetto, et al. (2009) quienes trabajaron con autoconciencia emocional, autorregulación emocional, motivación, empatía y competencias sociales. De manera similar Pérez, Bisquerra, Filella y Soldevila (2010) para la construcción de la escala consideraron 6 dimensiones (conciencia emocional, regulación emocional, autonomía emocional, competencias sociales y competencias para la vida y el bienestar, respectivamente). Estos estudios difieren sustancialmente con las dimensiones planteadas por Goleman (1995, 1998 y 2001) del cual menciona que la inteligencia emocional está compuesta por dos tipos de competencias: personales y/o emocionales (conciencia de uno mismo, autorregulación y motivación) y sociales (empatía y habilidades sociales). Para la presente escala se toman en cuenta las siguientes subdimensiones del constructo fueron establecidas por los siguientes autores: Conocimiento emocional (Bar On, 1997; Repetto y Pena, 2010; Goleman, 2001; Bisquerra y Pérez, 2007), siendo considerada como una competencia emocional, dado que es la capacidad para reconocer y comprender las emociones; regulación emocional considerada como una competencia emocional (Bar On, 1997; Repetto y Pena, 2010; Goleman, 2001; Bisquerra y Perez, 2007) puesto que es la capacidad para regular las propias emociones; capacidad participativa calificada como una competencia social (Stoner, 1996; Bunk, 1994; Alcañiz y Perez, 2008), de los cuales difiere su término dado por Repetto y Pena (2010) y Alles (2008), quienes definen la subdimensión trabajo en equipo como una competencia, no obstante, se modificó el término por capacidad participativa para definirla como una competencia social que facilita el trabajo en equipo, mejorando la productividad y la satisfacción laboral; empatía es también considerada una competencia social (Goleman, 
2001; Repetto y Pena, 2010; Bar On, 1977) ya que es considerada como la capacidad de reconocer, entender, comprender y ponerse en la situación de la otra persona, finalmente resolución de conflictos se considera una competencia social (Goleman, 2001; Repetto y Pena, 2010; Marcuello, 1999; Bisquerra, 2002) puesto que es la capacidad para negociar y dar solución a conflictos y controversias.

Teniendo en cuenta lo expuesto, la presente investigación define este constructo como conjunto de habilidades que permiten relacionarse con los demás y con uno mismo, reconociendo, manejando y expresando de manera correcta las emociones, de tal manera que promoverá el bienestar intrapersonal, y que, a su vez, se manifiesta en diferentes conductas interpersonales de manera eficaz.

Por consiguiente, el constructo de competencias socioemocionales está enmarcado dentro del área laboral, donde se considera como un aspecto importante de las habilidades para la empleabilidad. En el mundo laboral se acepta que la productividad depende de una fuerza de trabajo que sea socioemocionalmente competente (Frederickson, Petrides y Simmonds, 2012; Repetto, 2012 y Alles, 2008), entonces, esta investigación constituye el primer intento de abordar y medir este constructo de la realidad peruana. Asimismo, una de las contribuciones del presente estudio es la de promover un instrumento adaptado, válido y confiable. En lo que concierne a las propiedades psicométricas de este instrumento, cabe mencionar que, si bien es cierto que existen evidencias de validez y confiabilidad reportadas en varios estudios para diversas poblaciones en el extranjero, en nuestro entorno no encontramos antecedentes, aun cuando en la práctica laboral es conocido.

En lo que concierne a la validez, cabe señalar que su determinación está asociado a un conjunto de estudios encaminados a mostrar evidencias o avales científicos sobre si un instrumento mide realmente lo que se propone medir, como sostiene Aragón (2004) y Elousua (2003), la validez brinda el grado de adecuación y significancia a partir de las puntuaciones del test, y que dicha validez se determina a través de fuentes internas como externas.

En congruencia con lo mencionado, resulta importante destacar que la escala de competencias socioemocionales reúne las suficientes evidencias de validez, las fuentes de evidencia interna se obtuvieron a través del análisis de contenido (validez de contenido por criterio de jueces), la consistencia de la estructura interna del instrumento y la dimensionalidad del constructo (validez de constructo) mediante el análisis factorial.
En relación a la validez de contenido, los resultados muestran que más de la mayoría de los ítems alcanzan niveles adecuados de significación estadística en cuanto a la claridad, congruencia, contexto y dominio de los reactivos, para cuantificar los resultados se utilizó el coeficiente V de Aiken (Escurra, 1988), encontrando que los valores oscilan entre 1 y p $<0.01$, es importante mencionar que el ítem 10 presentó serias observaciones en cuanto a la claridad del constructo, es decir, que el ítem presenta dificultad para ser entendida, por lo cual siguiendo las sugerencias de los jueces se eliminó el ítem. Asimismo, los ítems 1, 2,3, 4, 5, 6 presentan observaciones por parte de uno de los jueces en cuanto a la congruencia y dominio del constructo, considerando las observaciones se modificó las imágenes haciéndolas más comprensibles para la aplicación de la escala, los ítems 5 y 6 se presentan observaciones en cuanto a la claridad y la congruencia, del mismo modo se tomó en cuenta las observaciones, se modificó el enunciado y se mejoró la imagen, el ítem 7 presenta observaciones en cuanto a claridad, considerando las observaciones la pregunta del ítem 7 ' Soy capaz de comprender mis emociones" fue modificado por "Soy capaz de comprender lo que siento ante diferentes situaciones".

Para evaluar la calidad métrica, se obtuvieron varios indicadores psicométricos para los ítems y se retuvo solamente aquellos ítems que cumplieron simultáneamente los siguientes requerimientos: 1) correlación ítem-sub dimensión mayor a 0.3; 2) coeficiente alpha crombach (al eliminar el ítem) igual o inferior al de la subdimensión y 3) saturación factorial en su dimensión por encima de 0.30 .

Para la subdimensión conocimiento emocional los ítems 7, 8, 9 y 10 fueron excluidos, debido a que no presenta un peso factorial mayor a .30. Al analizar la subdimensión regulación emocional los ítems 35 y 41 se eliminan por no tener correlación ítem - test y por tener un peso factorial menor de .30, así como también el ítem 36 es excluido por presentar una fiabilidad mayor a la de su dimensión. En la subdimensión capacidad participativa los ítems 43 y 11 se excluyen por presentar una fiabilidad mayor a la de su dimensión, así como también el ítem 44 es descartado por no tener correlación ítem - test, también los ítems 45, 46, 12, 13, 14, 17 ya que no tienen relación con la dimensión a medir. Además, en la subdimensión empatía los ítems 23, 55 y 56 no se consideraron por presentar una fiabilidad mayor a la de su dimensión, el ítem 22, 59 y 50 se descartaron porque su correlación ítem - test es menor al nivel estándar esperado (.30), también fueron eliminados los ítems 24, 25, 26, 57 y 58 por no ingresar teóricamente a la dimensión que 
les corresponde. Por último, para la subdimensión resolución de conflictos se eliminaron los ítems 30 y 32 por presentar una fiabilidad mayor a la de su dimensión, asimismo, los ítems 53 y 54 se eliminaron por no presentar una correlación ítem - test es decir que no mide teóricamente lo que se pretende medir y al mismo tiempo tener un peso factorial menor de .30.

Posteriormente, para la validación del constructo se realizó el análisis factorial exploratorio, donde se utilizó el método de estimación de componentes principales y método de rotación varimax para 5 factores fijos, donde el KMO presentó un valor de 0.782 , lo que indica que la correlación entre las subdimensiones es mediana, indicando la posibilidad para el uso del análisis factorial como lo propone García y Musito (1999) y Tomas y Oliver (2004). Así, al analizar el componente de capacidad participativa, se verificó que la mayoría de los ítems ingresan en el componente al que le corresponde con un peso factorial alto. Sin embargo, no ocurre con todos los ítems, como el 38 (regulación emocional) que ingresa a una dimensión que teóricamente no le corresponde (competencia participativa), no obstante, tiene peso factorial considerable en su dimensión, por tanto, se toma la decisión de mantener el ítem en su dimensión original. Asimismo, al analizar el componente empatía, se evidenció que los ítems 21 y 20 (competencia participativa) ingresa a una dimensión que teóricamente no le corresponde, sin embargo, tiene peso factorial considerable en su dimensión, por lo que se le mantiene en su misma dimensión.

Por último, al analizar el componente de regulación emocional, se observa que el ítem 52 (resolución de conflictos) ingresa a esta dimensión que teóricamente no le corresponde, no obstante, presenta un peso factorial considerable, por lo que se decide mantener en su propia dimensión.

Finalmente, se obtuvo como resultado 2 dimensiones: competencia emocional y competencia social que muestra adecuada correlación, los cuales se sostienen en el marco teórico propuesto (Goleman, 2001; Repetto y Pérez-González, 2007; Bar On, 2006; Repetto y Pena, 2010; Bisquerra y Perez, 2007; Stoner, 1996; Bunk, 1994; Alcañiz y Perez, 2008;
Alles, 2008; Marcuello, 1999; Bisquerra, 2002), asimismo, se obtuvieron 5 factores tales como: 1) capacidad participativa, 2) conocimiento emocional, 3 ) resolución de conflictos, 4) empatía y 5) regulación emocional. La varianza explica que la escala de competencias socioemocionales presenta un valor de $44,87 \%$, es decir, cumple con el nivel de varianza recomendado que es más del 40\% (Espinoza, 2008).

La fiabilidad global de la escala y de sus dimensiones se valoró calculando el índice de consistencia interna, mediante el coeficiente de alfa de Cronbach. La Escala de competencias socioemocionales presenta un nivel alto de fiabilidad (.789), de igual manera para las 2 dimensiones generales, obteniendo para la dimensión de competencia emocional (.532) y la competencia social (.795); asimismo, para las subdimensiones: conocimiento emocional (.709), regulación emocional (.603), capacidad participativa (.729), empatía (.497) y resolución de conflictos (.727). la subdimensión es considerada, según autores (Balart, 2013; Fernández, López y Marquéz, 2008; Olivera, 2010; López, Filippetti y Richaud, 2014) como un constructo amplio y complejo, por lo que muestra una medición con puntaje bajo (.497),por lo que dicha diemensión cuenta con pocos ítems (4), no logrando medir, en su totalidad, lo que se quiere medir.

Finalmente, se concluye que la escala de Competencias Socioemocionales (ECSE) es un instrumento fiable y válido que puede ser utilizado en nuestra realidad sociocultural.

\section{Declaración de financiamiento y de conflicto de intereses:}

El estudio fue financiado por los autores, quienes declaran no tener algún tipo de conflicto de interés en la investigación realizada.

\section{Correspondencia:}

Kiara Shalom Rojas Aliga

Colegio Unión. Universidad Peruana Unión, Carretera Central Km 19, Ñaña, Perú. Tel.: +51944576683.

E-mail: kiara_roal@hotmail.com/nayelihuamani@ outlook.com 


\section{REFERENCIAS BIBLIOGRÁFICAS}

Abad, F., Garrido, J. y Ponsoda, V. (2006). Introducción a la psicometría: teoría clásica de los test y teoría de la respuesta al ítem. Madrid: La Católica. Recuperado de: http://aprendeenlinea.udea.co/Ims/investigacion/ file.php/39/ARCHIVOS_2010/PDF/Intpsicometria_ aristidesvara_1_-pdf.

Alcañíz, M y Pérez, A. (2008). Las competencias transversales en una titulación de corte cuantitativo. Girona: Barcelona. Recuperado de: http://dugi-doc.udg. edu/bitstream/handle/10256/1032/153.pdf?sequence=1

Alles, M (2008). Desarrollo del talento humano. México: Gránica.

Bar-On, R. (1997). Development of the Baron EQ-I; A measure of emotional and social intelligence. Recuperado de: www.intechopen.com/download/ pdf/36451

Becerra, A. y La Serna, K. (2010). Las competencias que demanda el mercado laboral de los profesionales del campo económico-empresarial en la actualidad. Centro de Investigación de la Universidad del Pacífico. Recuperado de: http://srvnetappseg.up.edu.pe/ siswebciup/Files/DD1005\%20-\%20Becerra_La\%20 Serna.pdf

Bisquerra, R. (2002). La conciencia emocional. En Álvarez, A., Bisquerra, R., Manual de Orientación y tutoría (69-83). Barcelona: Praxis. Recuperado de: http://www.mediacioneducativa.com.ar/articulos/ colaboraciones/148-la-competencia-emocional.

Bisquerra, R. y Pérez, N. (2007). Las competencias emocionales. Educación XXI, 10, 61-82. Recuperado de: http://www.ub.edu/grop/catala/wp-content/ uploads/2014/03/Las-competencias-emocionales.pdf

Bunk, G. P. (1994). La transmisión de las competencias en la formación y el perfeccionamiento profesionales de la RFA. Revista Europea de Formación Profesional, 1, 8-14.

Cantero, M., Pérez, N. y Pérez, A. (2008). Diferencias en el perfil de competencias personales y socioemocionales en estudiantes universitarios de ciencia y educación. SUMMA, 5(1), 33-44. Recuperado de: dialnet.unirioja. es/descarga/articulo/2683135.pdf

Cantero, P. (2012). Competencias socio-emocionales en la inserción laboral del egresado universitario. (Tesis inédita de doctorado). Recuperado de: http://www. redalyc.org/pdf/706/70618742010.pdf

Cohn, B.; Merrell, K.; Felver-Grant, J.; Tom, K. y Endrulant, N. (2009). Strength-Based Assessment of social an emotional functioning: SEARS-C and SEARS-A. Boston: The National Association of School Psychologists. Recuperado de: http://strongkids. uoregon.edu/SEARS/Cohn2009.pdf

Costa, M. (2003). Competencia Socioemocional: una asignatura pendiente. Recuperado de: http:// www.cfieavila.com/jornadas $\% 20$ salud/salud/ CompetenciaSocioEmicional.htm
Fernández, M. (2011). Competencias socioemocionales en adolescentes de altas habilidades: un estudio comparativo. (Tesis inédita de doctorado). Universidad de Murcia, España. Recuperado de: www.tdx.cat/ handle/10803/38356

Fragoso, R. (2015). Inteligencia emocional y competencias emocionales en educación superior, ¿un mismo concepto? UNIVERSIA, 16(6), 110-125. Recuperado de:https://ries.universia.net/article/viewFile/1085/1514

Goleman, D. (1995). Inteligencia emocional. Barcelona: Kairos.

Goleman, D. (1999). La práctica de inteligencia emocional. Barcelona: Kairos.

Gonzáles, S. (2014). Competencias socioemocionales en alumnado de primaria: validación inicial de la escala sears-c. (Trabajo de fin de grado Uva, Universidad de Valladolid) Recuperado de: https://uvadoc.uva.es/ bitstream/10324/7747/1/TFG-G\%20862.pdf

Manpower Group (2013). Estudio Manpower group sobre escases de talento. Recuperado de: https:// candidate.manpower.com/wps/wcm/connect/ ESCampus/e1109289-7b5e-4609-9bd2-45c0cb5f640b/ Estudio+ManpowerGroup+Escasez+Talento+2013. pdf?MOD=AJPERE

Marcuello, A. (1999). Las competencias sociales, conceptos y técnicas para su desarrollo. Recuperado de: Http:// www.Psicología-online.com/autoayuda/asertividad/ competencias_sociales.shtml.

Mayer, J. y Salovey, P. (1997). Whay is emotional intelligence. In Salovey P, \& Sluyter (Eds.). Emotional development literacy and Emotional Intelligence: implications for educator. New York: Basic Books Recuperado de: http://www.unh.edu/emotional_ intelligence/EI\%20Assets/Reprints...EI\%20Proper/ EI1997MSWhatIsEI.pdf

Molero, D., Belchi-Reyes, M., Torres-Luque, G. (2012). Competencias socioemocionales en practicantes de deportes de montaña. Scientific Section Martos (Spain), 4(2) ,199-208. Recuperado de: www.elsevier. com/locate/paid

Nunnally, J. (1970) Introducción a la medición psicológica. "Reseña histórica de la medición psicológica”. Buenos Aires: Paidós.

Organización Internacional del Trabajo (2013). Tendencias mundiales del empleo juvenil 2013. Oficina Internacional del trabajo. Recuperado de: http://www. ilo.org/wcmsp5/groups/public/---dgreports/---dcomm/ documents/publication/wcms_222658.pdf

Pérez, J. (2003) Sobre la validez de constructo de la inteligencia emocional. Encuentros en la psicología social, 1(2), 252-257.

Pérez, J. C., Petrides, K. V., \& Furnham, A. (2005). Measuring Trait Emotional Intelligence. En Schulze 
R. and Roberts R. D. (Eds.) International Handbook of Emotional Intelligence, Cambridge, 181-201.

Pérez, N., Bisquerra, R., Filella, G. y Soldevila, A. (2010). Construcción del cuestionario de desarrollo emocional de adultos (QDE-A). Revista Española de Orientación y psicopedagogía, 21(2), 367-379. Recuperado de: http://www.uned.es/reop/pdfs/2010/21-2\%20-\%20 Perez\%20Escoda\%20-\%20Bisquerra.pdf

Quintero, A. (2004). Selección de personal por competencias laborales disponible en Enlaces Selcom: El Salvador. Recuperado de: http://www.uv.es/ selva/ gestion/articles/selcompe.htm

Repetto, E. y Pena, M. (2010). Las competencias socioemocionales como factor de calidad en la educación. RINACE, 8(5), 82-95. Recuperado de: http://dialnet. unirioja.es/servlet/articulo?codigo $=3921000$

Repetto, E., Pena, M., Mudarra, M. y Uribarri, M. (2009). Orientación de las competencias socioemocionales de los alumnos de Educación Secundaria en contextos multiculturales. Revista Electrónica de Investigación Psicoeducativa, 5(1), 159-178. Recuperado de: http:// www.uned.es/andresbello/documentos/mario.pdf

Repetto, E., Pérez, J. (2007). Formación de competencias Socioemocionales a través de la práctica en empresas. 40(1), 92-112, ISSN 19770235. Recuperado de: http//:dialnet.unirioja.es/servlet/autor?
Repetto, E., Beltrán, G., Garay, A., Pena, M. (2006). Validación del Inventario de Competencias Socioemocionales en un grupo de 375 estudiantes de ciclos formativos y universitarios. Revistauned, 17(2), 213-223. Recuperado de: http://www.uned.es/reop/ pdfs/2006/17-2-2\%20-\%20Elvira\%20Repetto.pdf

Sainz, M. (2010) Creatividad, personalidad y competencia socioemocional en alumnos de altas habilidades versus no altas habilidades. Tesis Doctoral. Universidad de Murcia. Recuperado de: http://www.redalyc.org/ articulo.oa?id=217022109008

Sainz, M.; Soto, G.; Ferrándiz, C.; Almeida, L.; Fernández, M. y Ferrando, M. (2011). Competencias socioemocionales y creatividad según el nivel de inteligencia. REDALYC, 14(3). 97-106. Recuperado de: http://www. redalyc.org/articulo.oa?id=217022109008

Senra, M.; Pérez, J. y Manzano, N. (2007). Competencias socioemocionales y alcoholismo en mujeres: revisión y estado actual de la cuestión. Revista Española de Orientación y Psicopedagogía, 18(1), 73-82. Recuperado de: http://www.uned.es/reop/ pdfs/2007/18-1\%20-\%20Maria\%20Senra.pdf

Recibido: 06/07/2016 Aceptado: 15/08/2016 